\title{
Identification and Characterization of a Selective Human Carbonyl Reductase 1 Substrate ${ }^{\text {S }}$
}

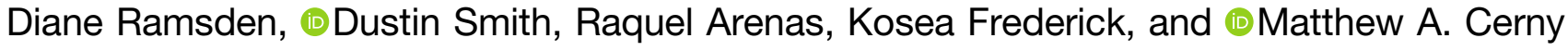 \\ Drug Metabolism and Pharmacokinetics, Boehringer Ingelheim Pharmaceuticals, Inc., Ridgefield, Connecticut
}

Received May 18, 2018; accepted July 26, 2018

\begin{abstract}
During drug discovery efforts targeting inhibition of cytochrome P450 11B2 (CYP11B2)-mediated production of aldosterone as a therapeutic approach for the treatment of chronic kidney disease and hypertension, (S)-6-(5-fluoro-4-(1-hydroxyethyl)pyridin-3-yl)3,4-dihydro-1,8-naphthyridine-1(2H)-carboxamide (1) was identified as a potent and selective inhibitor of CYP11B2. Preclinical studies characterized 1 as low clearance in both in vitro test systems and in vivo in preclinical species. Despite low metabolic conversion, an active ketone metabolite (2) was identified from in vitro metabolite-identification studies. Due to the inhibitory activity of 2 against CYP11B2 as well as the potential for it to undergo reductive metabolism back to 1 , the formation and elimination of 2 were characterized and are the focus of this manuscript. A series
\end{abstract}

of in vitro investigations determined that 1 was slowly oxidized to 2 by cytochrome P450s 2D6, 3A4, and 3A5, followed by stereoselective reduction back to 1 and not its enantiomer (3). Importantly, reduction of 2 was mediated by an NADPH-dependent cytosolic enzyme. Studies with human cytosolic fractions from multiple tissues, selective inhibitors, and recombinantly expressed enzymes indicated that carbonyl reductase 1 (CBR1) is responsible for this transformation in humans. Carbonyl reduction is emerging as an important pathway for endogenous and xenobiotic metabolism. With a lack of selective substrates and inhibitors to enable characterization of the involvement of CBR1, 2 could be a useful probe to assess CBR1 activity in vitro in both subcellular fractions and in cell-based systems.

\section{Introduction}

The predominant mineralocorticoid, aldosterone, controls salt and water balance via binding to the mineralocorticoid receptor. Aldosterone actions have also been proposed to occur via other so-called nongenomic pathways (Funder, 2001; Boldyreff and Wehling, 2003; Vinson and Coghlan, 2010; Dooley et al., 2012). Although the exact mechanism(s) is not currently understood, elevated levels of aldosterone are associated with a number of diseases (i.e., chronic kidney disease, hypertension, and obesity) (Hwang et al., 2013). The renin-angiotensin aldosterone system has been the focus of much attention by the pharmaceutical industry and has resulted in multiple drug classes which include direct renin inhibitors, angiotensin-converting enzyme inhibitors, angiotensin receptor blockers, and mineralocorticoid receptor antagonists. Direct inhibition of the production of aldosterone offers a novel means of treating the diseases indicated earlier and may have the additional benefit of decreasing the interaction of aldosterone through both genomic and nongenomic pathways (Cerny, 2013; Hu et al., 2014; Brem and Gong, 2015; Oparil and Schmieder, 2015).

Drug discovery efforts (Cerny et al., 2015; Weldon et al., 2016) identified 1 [ $(S)$-6-(5-fluoro-4-(1-hydroxyethyl)pyridin-3-yl)-3,4dihydro-1,8-naphthyridine-1(2H)-carboxamide; Fig. 1) as a potent inhibitor of aldosterone synthase (CYP11B2). Additionally, 1 only weakly inhibits the highly similar enzyme CYP11B1, as well as other steroidogenic and

https://doi.org/10.1124/dmd.118.082487.

S This article has supplemental material available at dmd.aspetjournals.org. xenobiotic-metabolizing cytochrome P450s. During characterization the absorption, distribution, metabolism, and excretion properties of $\mathbf{1}$, a ketone metabolite (2) was identified in incubations with liver microsomes and hepatocytes as well as in recombinantly expressed cytochrome P450 (P450) enzymes. Although the abundance of 2 was found to be relatively low in these systems, additional in vitro characterization of the metabolism of $\mathbf{2}$ was undertaken for the following reasons: 1) 2 was shown to have inhibitory properties toward CYP11B2 similar to that of $\mathbf{1}$; 2) reductive metabolism of $\mathbf{2}$ may result in generation of either $\mathbf{1}$ or its enantiomer, $\mathbf{3}$ (Fig. 1), therefore requiring chiral chromatographic analyses of clinical samples; and 3) reversible metabolism of $\mathbf{2}$ may contribute to the low clearance observed for $\mathbf{1}$. Based on the results of the studies described herein, $\mathbf{2}$ was determined to be a selective substrate of human carbonyl reductase 1 (CBR1).

Carbonyl reduction has emerged as an important non-P450 pathway which contributes to the metabolism of both endogenous substrates and xenobiotics (Málatková and Wsól, 2014; Cerny, 2016). These reactions can be catalyzed by the aldo-keto reductase (AKR) and carbonyl reductase family of enzymes, and substrate overlap between these families of enzymes is often observed. NAD $(\mathrm{P}) \mathrm{H}$-dependent enzymes of both the AKR and short-chain dehydrogenase/reductase (SDR) families of enzymes have been shown to carry out these reactions. CBRs (EC. 1.1.1.184) represent a subfamily of SDR enzymes that includes three isoforms in humans: CBR1, human carbonyl reductase 3 (CBR3), and human carbonyl reductase 4 (CBR4). Whereas CBR1 and CBR3 are both cytosolic enzymes, CBR4 is found in the mitochondria. CBR3 and CBR4 have been shown to have low carbonyl-reducing

ABBREVIATIONS: AKR, aldo-keto reductase; CBR1, human carbonyl reductase 1; CBR3, human carbonyl reductase 3; CBR4, human carbonyl reductase 4; $\mathrm{Cl}_{\text {int, }}$, intrinsic clearance; CR, carbonyl reductase; HLCyt, human liver cytosol; HLM, human liver micrsome; LC/MS/MS, liquid chromatography coupled with tandem mass spectrometry; LCyt, liver cytosol; LM, liver microsomes; P450, cytochrome P450; rP450, recombinant cytochrome P450; SDR, short-chain dehydrogenase/reductase. 
<smiles>CC(O)c1c(F)cncc1-c1cnc2c(c1)CCCN2C(N)=O</smiles>

1<smiles>CC(=O)c1c(F)cncc1-c1cnc2c(c1)CCCN2C(N)=O</smiles>
2
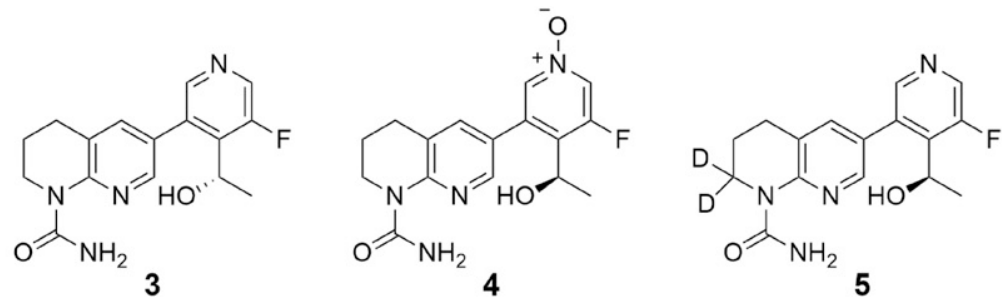

Fig. 1. Structures of compounds 1-5 activity or narrow substrate specificity. In contrast, CBR1 has been shown to be the most important enzyme of carbonyl-reducing enzymes, as it is involved in the metabolism of multiple clinically important drugs, including doxorubicin, daunorubicin, nabumetone, loxoprofen, haloperidol, pentoxifylline, and bupropion (Rosemond and Walsh, 2004). Despite the recognition that this pathway is important to the metabolism of carbonyl-containing compounds, there is still a paucity of data in the literature, and further research is needed. Identification of $\mathbf{2}$ as a selective substrate for CBR1 may serve as a useful probe for further study of this enzyme and its involvement in the metabolism of drugs and endogenous substances.

\section{Materials and Methods}

Reagents. $\beta$-Nicotinamide adenine dinucleotide phosphate reduced $(\mathrm{NADPH}) ; \beta$-nicotinamide adenine dinucleotide, reduced disodium salt hydrate (NADH); dimethylsulfoxide; acetonitrile $(\mathrm{MeCN})$; phenobarbital; zopolrestat; phenolphthalein; flufenamic acid; chenodeoxycholic acid; medroxyprogesterone 17-acetate; dexamethasone; indomethacin; menadione; quercetin ethacrynic acid; dicumarol; 4-methyl pyrazole; and disulfiram were purchased from MilliporeSigma (St. Louis, MO). Compounds 1-5 were from the Boehringer Ingelheim (Ridgefield, CT) compound collection and were prepared by published methods (Balestra et al., 2014). Pooled liver microsomes from mixed-gender human (HLM), pooled cynomolgus male monkey, pooled male beagle dog, and pooled male Wistar Han rat were purchased from Corning (Tewksbury, MA). Pooled mixed-gender human, cynomolgus male monkey, male beagle dog, and male Wistar Han rat liver, kidney, and lung cytosolic fractions were purchased from Corning. Pooled human, male cynomolgus monkey, male beagle dog, and male Wistar Han rat suspension hepatocytes were obtained from Bioreclamation IVT (Baltimore, MD). Recombinant CBR1, CBR3, and CBR4 were purchased from Abcam (Cambridge, MA).

Metabolite-Identification Studies in Liver Microsomes. Compound 1 $(10 \mu \mathrm{M})$ was incubated with human, monkey, dog, and rat liver microsomes (LM) at a $1-\mathrm{mg} / \mathrm{ml}$ final protein concentration in $0.1 \mathrm{M}$ phosphate buffer $(\mathrm{pH} 7.4)$ for 2 hours at $37^{\circ} \mathrm{C}$ in a total volume of $1 \mathrm{ml}$ using either NADPH or NADH $(1 \mathrm{mM})$ as a cofactor. Additional metabolite-identification studies were carried out on $2(10 \mu \mathrm{M})$ in both HLM and human liver cytosol (HLCyt) as described earlier for 1 . Incubations were halted by addition of cold $\mathrm{MeCN}(1 \mathrm{ml})$ followed by thorough vortexing and removal of precipitate protein by centrifugation (10 minutes at $3000 \mathrm{~g}$ ). Supernatants were removed and concentrated to dryness under a stream of nitrogen. The resulting material was then reconstituted in a starting mobile phase and analyzed by liquid chromatography coupled with tandem mass spectrometry (LC/MS/MS) as described herein.

Metabolite-Identification Studies Using Hepatocytes. Compound 1 $(10 \mu \mathrm{M})$ was incubated with hepatocytes (final concentration 2 million cells $/ \mathrm{ml}$ ) in Williams' $\mathrm{E}$ medium for 4 hours at $37^{\circ} \mathrm{C}$ in a total volume of $0.5 \mathrm{ml}$. Incubations were halted by addition of cold $\mathrm{MeCN}(1 \mathrm{ml})$ followed by thorough vortexing and removal of precipitate protein by centrifugation (10 minutes at $3000 \mathrm{~g}$ ). Supernatants were removed and concentrated to dryness under a stream of nitrogen. The resulting material was then reconstituted in a starting mobile phase and analyzed by LC/MS/MS as described herein.

Reaction Phenotyping Studies. Individual recombinant cytochrome P450 isoforms (rP450s; $50 \mathrm{pmol} / \mathrm{ml}$ final concentration) were incubated with $\mathbf{1}(1 \mu \mathrm{M})$ in $0.1 \mathrm{M}$ phosphate buffer ( $\mathrm{pH}$ 7.4) containing magnesium chloride ( $3 \mathrm{mM}$ final concentration) at $37^{\circ} \mathrm{C}$. Incubations were started by the addition of NADPH
( $1 \mathrm{mM}$ final concentration), bringing the total volume to $0.4 \mathrm{ml}$. Aliquots $(50 \mu \mathrm{l})$ were removed at $0,5,10,20$, and 30 minutes and added to cold $\mathrm{MeCN}(150 \mu \mathrm{l})$ containing internal standard. Quenched samples were submitted to thorough vortexing and removal of precipitate protein by centrifugation (10 minutes at $3000 \mathrm{~g}$ ). Supernatants were removed and concentrated to dryness under a stream of nitrogen. The resulting material was then reconstituted in a starting mobile phase and analyzed by LC/MS/MS as described herein.

Kinetics Assessment of Ketone Reduction. Formation of $\mathbf{1}$ from $\mathbf{2}$ was assessed in triplicate incubations in $0.1 \mathrm{M}$ potassium phosphate buffer ( $\mathrm{pH}$ 7.4) containing varying concentrations of 2 [12 concentrations between 0 and 100 for liver cytosol (LCyt) and HLM or 0-500 $\mu \mathrm{M}$ for CBR1, CBR3, and CBR4]; cytosolic or microsomal fractions $(1 \mathrm{mg} / \mathrm{ml}$ final protein concentration) from human or other preclinical species or recombinant CBR1, CBR3, or CBR4 $(0.01 \mathrm{mg} / \mathrm{ml}$ final protein concentration $)$; and NADPH (1 mM final concentration) at a final volume of $0.12 \mathrm{ml}$. For specified experiments, NADPH was replaced with NADH (1 mM final concentration). For all experiments, incubations were preincubated for 5 minutes and started by the addition of cofactor. After 5 minutes, the incubations were quenched by the addition of $\mathrm{MeCN}(0.12 \mathrm{ml})$ containing internal standard. Precipitated proteins were removed by centrifugation ( 5 minutes at $3000 \mathrm{~g}$ ). Samples were analyzed by LC/MS/MS as indicated herein.

Substrate Depletion in Cytosolic Fractions across Species. Parent disappearance experiments were conducted in triplicate (human) or quadruplicate (monkey, dog, or rat) essentially as described earlier. For these experiments, a single $1 \mu \mathrm{M}$ concentration of $\mathbf{2}$ was used as substrate, and incubations were terminated as described earlier at $0,5,15,30$, and 60 minutes. Precipitated proteins were removed by centrifugation (10 minutes at $3000 \mathrm{~g}$ ). Samples were analyzed by LC/MS/MS as indicated herein.

Inhibition of the Reduction of $\mathbf{2}$ to 1 in Human Liver Cytosol. The effect of reductase inhibitors was assessed by incubation of $2(15 \mu \mathrm{M})$ with HLCyt $(1 \mathrm{mg} / \mathrm{ml}), 0.1 \mathrm{M}$ potassium phosphate buffer $(\mathrm{pH} 7.4)$, and NADPH in the presence of 10 or $100 \mu \mathrm{M}$ of the following inhibitors: phenobarbital, zopolrestat, phenolphthalein, flufenamic acid, chenodeoxycholic acid, medroxyprogesterone 17-acetate, dexamethasone, indomethacin, menadione, quercetin, ethacrynic acid, dicumarol, 4-methyl pyrazole, and disulfiram. Incubations were started by the addition of NADPH and warmed at $37^{\circ} \mathrm{C}$ for 5 minutes. Incubations were quenched by the addition of $\mathrm{MeCN}$ containing internal standard. Samples were analyzed by LC/MS/MS as indicated herein.

\section{LC/MS/MS Analyses}

Metabolite Identification. Samples $(20 \mu \mathrm{l})$ were injected on a Supelco Discovery C18 $5 \mu 2.1 \times 150$-mm column maintained at room temperature using a Thermo Accela High Speed LC system (ThermoFisher Scientific, Waltham, MA). A solvent system consisting of water with $0.1 \%$ formic acid (mobile phase $\mathrm{A}$ ) and acetonitrile with $0.1 \%$ formic acid (mobile phase B) at a flow rate of $0.4 \mathrm{ml} / \mathrm{min}$ was used with the following gradient: $0-5$ minutes, $15 \% \mathrm{~B} ; 5-10$ minutes, $15 \%-52.5 \% \mathrm{~B}$; 15-16 minutes, $52.5 \%-85 \% \mathrm{~B}$, maintained $85 \%$ B for 2 minutes; 18-19 minutes, $15 \% \mathrm{~B}$, then equilibrated for 3 minutes. The column eluent was analyzed by MS on a Thermo LTQ OrbiTrap XL Mass Spectrometer (ThermoFisher Scientific) run in positive mode using Fourier Transform Mass Spectrometry and data-dependent Fourier Transform MS/MS scans (Collision-induced dissociation and Higherenergy collisional dissociation) to elucidate metabolite structures. An 
in-line Accela photodiode array detector (Thermo Scientific) obtained simultaneous UV absorption spectra. The resulting data were analyzed using Xcalibur 3.0 and Thermo Metworks 1.3 software (ThermoFisher Scientific).

Analysis of Kinetics Study Samples. Samples $(5 \mu \mathrm{l})$ were injected on a Phenomenex (Torrance, CA) Kinetex C18 $1.7 \mu \mathrm{m}, 2.1 \times 50 \mathrm{~mm}$ column maintained at $40^{\circ} \mathrm{C}$ using a Waters (Milford, MA) Acquity I-Class UPLC System. A solvent system consisting of water with $0.1 \%$ formic acid (mobile phase A) and acetonitrile with $0.1 \%$ formic acid (mobile phase B) at a flow rate of $0.5 \mathrm{ml} / \mathrm{min}$ was used with the following gradient: $0-0.50$ minutes, $10 \% \mathrm{~B} ; 0.50-1.50$ minutes, $10 \%-90 \% \mathrm{~B}$; $1.50-2.00$ minutes, maintained at $90 \% \mathrm{~B} ; 2.00-2.01$ minutes, $90 \%-10 \%$ $\mathrm{B}$; 2.01-2.50 minutes, re-equilibrate at $10 \% \mathrm{~B}$. The column eluent was analyzed by MS on an AB Sciex (Framingham, MA) API 6500 Q-Trap Mass Spectrometer (ThermoFisher Scientific) run in positive mode using the following multiple reaction monitoring method: for $\mathbf{1}, \mathrm{Q} 1 / \mathrm{Q} 3$ $317.2 \rightarrow 254$, declustering potential 60 , collision energy 25 ; for $\mathbf{2}$, Q1/Q3 315.1 $\rightarrow 252$, declustering potential 60, collision energy 25. A standard curve was constructed by serial dilution in a control tissue sample ranging from 10 to $0.001 \mu \mathrm{M}$. The resulting $\mathrm{MS}$ data were analyzed using Analyst 1.4.2 software (AB Sciex, Framingham, MA).

Chiral Chromatography of 1 and 3. Samples $(5 \mu \mathrm{l})$ were injected on a Chiral-AGP $2.0 \times 100 \mathrm{~mm}$ column (Chiral Technologies, Inc., West Chester, PA) maintained at room temperature using a Waters Acquity I-Class UPLC System. A solvent system consisting of water (mobile phase A) and acetonitrile (mobile phase B) at a flow rate of $0.25 \mathrm{ml} / \mathrm{min}$ was used with an isocratic flow of $95 \% \mathrm{~A}$ and $5 \% \mathrm{~B}$. The column eluent was analyzed by MS on an AB Sciex API 6500 Q-Trap Mass Spectrometer run in positive mode using the following multiple reaction monitoring method: for both $\mathbf{1}$ and 3, Q1/Q3 317.2 $\rightarrow 254$, DP 60, CE 25. The resulting MS data were analyzed using Analyst 1.4.2 software.

\section{Calculations}

Kinetics Assessment of Ketone Reduction. The rate of formation of $\mathbf{1}$ from $\mathbf{2}$ was plotted against the nominal incubation concentrations of $\mathbf{2}$ and fit to the Michaelis-Menten equation using GraphPad Prism 7.02 (GraphPad Software, La Jolla, CA) to determine the kinetics parameters $\left(\mathrm{K}_{\mathrm{m}}\right.$ and $\left.\mathrm{V}_{\max }\right)$. Given the low lipophilicity of $2(\log \mathrm{P}=1.7)$, nonspecific binding to microsomes is expected to be low. In vitro intrinsic clearance $\left(\mathrm{Cl}_{\text {int }}\right)$ was calculated from these parameters using eq. 1 :

$$
\mathrm{Cl}_{\mathrm{int}}=\frac{\mathrm{V}_{\mathrm{max}}}{\mathrm{K}_{\mathrm{m}}} .
$$

Substrate Depletion in Cytosolic Fractions across Species. The natural $\log$ of percentage of substrate remaining was plotted versus time to determine the rate of depletion $\left(\mathrm{k}_{\mathrm{deg}}\right)$. From these data, $\mathrm{Cl}_{\text {int }}$ was calculated as described in eq. 2 :

$$
\mathrm{Cl}_{\text {int }}=\mathrm{k}_{\mathrm{deg}} \times \frac{\mathrm{ml} \text { incubation }}{1 \mathrm{mg} \text { cytosolic protein }} .
$$

\section{Results}

Metabolite Identification. Metabolism studies on $\mathbf{1}$ were carried out across species in a number of in vitro systems, including LM, hepatocytes, and rP450s. In incubations of LM with 1 performed in the absence of NADPH, neither consumption of $\mathbf{1}$ nor formation of metabolites was observed. Generally, greater consumption of $\mathbf{1}$ was observed in LM than

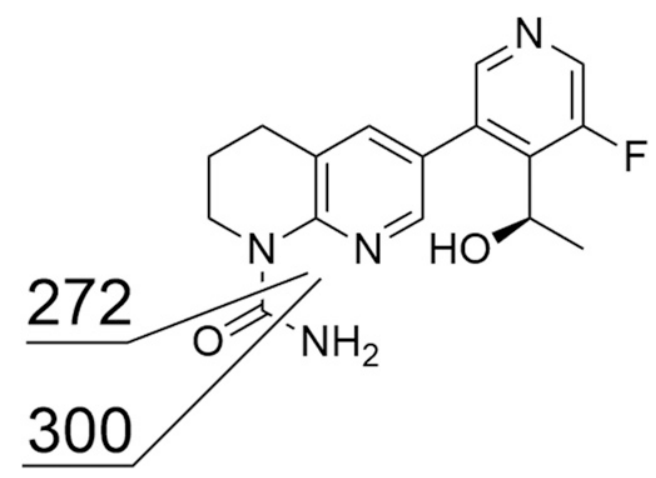

Fig. 2. Major MS fragments for compound 1 and metabolites.

in hepatocytes across species (Supplemental Tables S1 and S2). However, in both human LM and hepatocytes, low turnover $(<5 \%$ consumed in 60 minutes, Supplemental Fig. S1) was observed for 1. Greater consumption of $\mathbf{1}$ and formation of metabolites were observed in monkey, dog, and rat LM and hepatocytes compared with human. In samples from these incubations, $\mathbf{1}$ was shown to elute at 10.8 minutes with prominent fragmentation occurring through the urea moiety (Fig. 2), yielding fragments of $m / z, 300$ and 274 .

As shown in Fig. 3, three mono-oxygenated metabolites $(\mathrm{m} / \mathrm{z}, 333)$ of 1 were observed. As the prominent fragmentation for these metabolites occurs via the urea moiety, similar to what was described earlier for $\mathbf{1}$, little structural information concerning the position of metabolism was ascertained from MS/MS data. The identity of one of these three metabolites ( $t_{R}=9.2$ minutes $)$ was determined to be the pyridine $N$-oxide (4) based on a synthetic standard. The site of metabolism for a second $\mathrm{m} / \mathrm{z}$ 333 metabolite ( $\mathrm{m} 332 \_2, \mathrm{t}_{\mathrm{R}}=9.8$ minutes) was identified as being on the methylene adjacent to the urea nitrogen. This was established using a deuterated analog of $\mathbf{1}(\mathbf{5})$, where metabolism of 5 resulted in loss of one of the two deuterium atoms $(\mathrm{m} / \mathrm{z}, 334)$ and could result in formation of the ringintact hydroxyl species and/or the ring-opened aldehyde metabolite. The structure of the third $m / z, 333\left(\mathrm{~m} 332 \_3, \mathrm{t}_{\mathrm{R}}=10.5\right.$ minutes $)$ metabolite was not determined. Subsequent reduction and oxidation of the aldehyde form of m332_2 gave rise to both the $\mathrm{m} / \mathrm{z}, 335\left(\mathrm{~m} 334, \mathrm{t}_{\mathrm{R}}=8.0\right.$ minutes) and $\mathrm{m} / \mathrm{z}$ $349\left(\mathrm{~m} 348, \mathrm{t}_{\mathrm{R}}=8.5\right.$ minutes) metabolites, respectively, which are only observed in hepatocytes. The structures of both of these metabolites were confirmed with synthetic standards.

Another minor metabolite with $m / z, 315\left(t_{R}=12.9\right.$ minutes, $\left.-2 \mathrm{H}\right)$ was identified in a number of matrices. The structure of this metabolite was confirmed with a synthetic standard of $\mathbf{2}$, which matched the chromatographic retention and mass spectrometric fragmentation pattern of the $\mathrm{m} / \mathrm{z}$ 315 metabolite (data not shown). Follow-up studies determined 2 to be a potent and selective inhibitor of CYP11B2 $\left(\right.$ CYP11B2 $\mathrm{IC}_{50}=9.7 \mathrm{nM}$, CYP11B1 $\left.\mathrm{IC}_{50}=6200 \mathrm{nM}\right)$ using cynomolgus monkey adrenal homogenate assays (Cerny et al., 2015).

Metabolites observed in monkey, dog, and rat LM and hepatocytes corresponded to the metabolites observed in human matrices. No human unique metabolites were observed, and no additional metabolites were detected in preclinical species LM and hepatocytes. Metabolism of $\mathbf{1}$ in $\mathrm{rP} 450 \mathrm{~s}$ resulted in the formation of only oxidative metabolites (4, m332_2, m332_3, and 2), with CYP2C8, CYP2C9, CYP2C19, CYP2D6, CYP3A4, and CYP3A5 contributing to the metabolism of 1 (Supplemental Table S3).

Additional metabolite-identification studies were carried out on 2 in both HLM and HLCyt. In these studies, $\mathbf{2}$ was also found to be relatively stable in HLM and HLCyt incubations supplemented with NADH $(<10 \%$ consumed in 60 minutes). The major metabolite observed in 

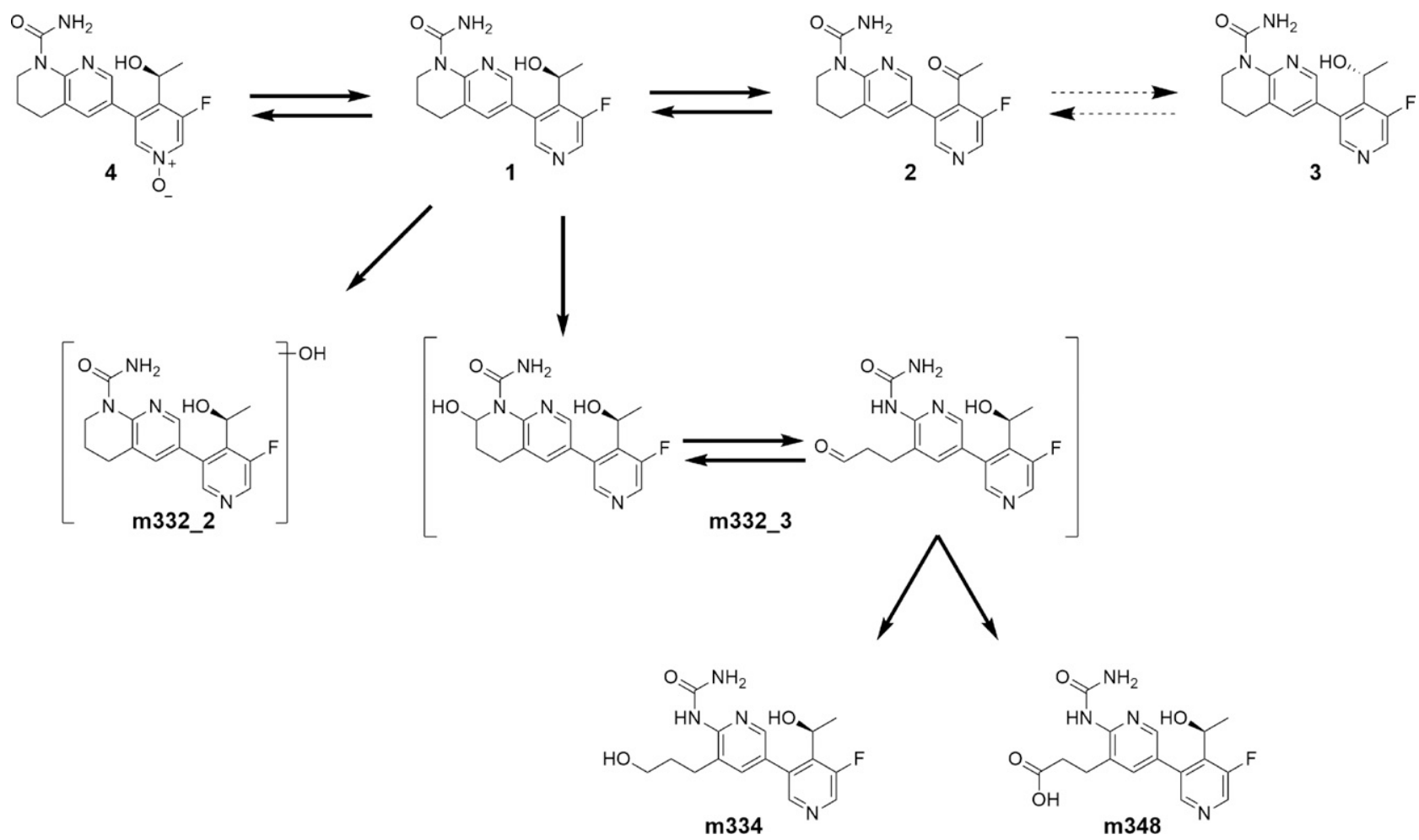

Fig. 3. Metabolic scheme for compound $\mathbf{1}$.

these studies was reduction of $\mathbf{2}$ to a metabolite consistent with the enantiomers, $\mathbf{1}$ and $\mathbf{3}$, in terms of mass, retention time, and fragmentation pattern and represents $2.2 \%$ and $5.1 \%$ of the total UV peak area, respectively (Supplemental Table S4). When $\mathbf{2}$ was incubated with HLM and NADPH, formation of a metabolite consistent with $\mathbf{1}$ or $\mathbf{3}$ was also

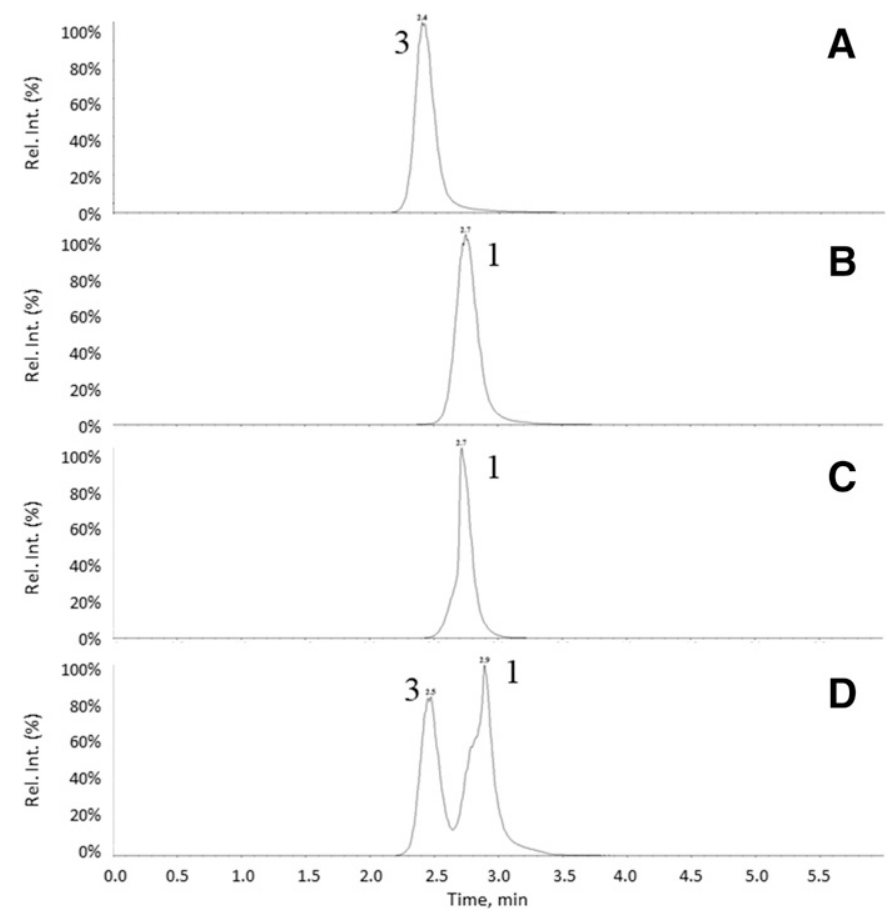

Fig. 4. Chiral chromatography of $\mathbf{3}$ (A), 1 (B), incubation of $\mathbf{2}$ with HLCyt + NADPH (C), and incubation of $\mathbf{2}$ with HLCyt spiked with 3 (D). Rel. Int. Relative Intensity. the major biotransformation, representing $\sim 45 \%$ of the UV peak area. A minor monohydroxylated metabolite of 2 with $\mathrm{m} / \mathrm{z} 331(0.6 \%$ of UV peak area) was also observed under these conditions as well as three other minor metabolites which were only detectable by MS. Incubation of 2 with HLCyt and NADPH resulted in significant formation of a metabolite consistent with $\mathbf{1}$ or $\mathbf{3}$, which represented almost $99 \%$ of the total UV peak area.

Determination of Stereoselectivity of the Reduction of 2 . As reduction of $\mathbf{2}$ may result in formation of either $\mathbf{1}$ and/or $\mathbf{3}$, chiral chromatography was developed to determine the stereoselectivity of this reduction. Chromatographic resolution of $\mathbf{1}$ from $\mathbf{3}$ was achieved as shown in Fig. 4, A and B. Subsequently, analysis of a sample of the HLCyt incubation of $\mathbf{2}$ supplemented with NADPH resulted in a single peak with a retention time that matched 1 (Fig. 4C). Spiking of this sample with $\mathbf{3}$ followed by chromatographic analysis resulted in two peaks, an earlier eluting peak corresponding to $\mathbf{3}$ which was not present in the incubation sample and the previously observed peak which corresponded to 1 (Fig. 4D). This analysis indicated that reductive metabolism of $\mathbf{2}$ occurs stereoselectively, resulting in conversion of $\mathbf{2}$ back to $\mathbf{1}$ with no or minimal formation of $\mathbf{3}$.

Kinetics Studies. To further characterize the reduction of $\mathbf{2}$ to $\mathbf{1}$, kinetics studies were carried out in human cytosolic fractions from multiple tissues as well as HLM and recombinant CBR1, CBR3, and CBR4. Initial incubations were run with both NADH and NADPH to determine the optimal cofactor for supporting this reductive transformation. As the determined solubility of $\mathbf{2}$ at physiologic $\mathrm{pH}$ was $\sim 100 \mu \mathrm{M}$, data generated at concentrations exceeding this limit were excluded from kinetics assessments. Kinetics of the reductive metabolism of $\mathbf{1}$ followed Michalis-Menten kinetics, and in general, similar $\mathrm{K}_{\mathrm{m}}$ values were observed across all matrices (Table 1). In both HLCyt and HLM, NADPH-fortified incubations displayed higher $\mathrm{Cl}_{\text {int }}$ for the reductive metabolism of $\mathbf{2}$ (Fig. 5; Table 1). Formation of $\mathbf{1}$ from 2 was observed in NADPH-fortified incubations of either CBR3 or CBR4 
TABLE 1

Enzyme kinetic parameters for conversion of $\mathbf{2}$ to $\mathbf{1}$ in various matrices supplemented with either NADPH or NADH

\begin{tabular}{|c|c|c|c|c|c|c|}
\hline Source & Tissue & Fraction & Cofactor & $\mathrm{K}_{\mathrm{m}}$ & $\mathrm{V}_{\max }$ & $\mathrm{Cl}_{\text {int }}$ \\
\hline & & & & $\mu M$ & $\mathrm{pmol} / \mathrm{min} / \mathrm{mg}$ & $\mu \mathrm{l} / \mathrm{min} / \mathrm{mg}$ protein \\
\hline Human CBR1 & & & NADPH & 107.6 & 1180 & 10.97 \\
\hline \multirow[t]{7}{*}{ Human } & Liver & Cytosol & NADPH & 91.2 & 15.8 & 0.17 \\
\hline & & Cytosol & NADH & 63.6 & 0.49 & 0.0078 \\
\hline & & Microsomes & NADPH & 52.3 & 1.68 & 0.032 \\
\hline & & Microsomes & NADH & 56.4 & 0.15 & 0.0027 \\
\hline & Intestine & Cytosol & NADPH & 39.8 & 7.38 & 0.19 \\
\hline & Lung & Cytosol & NADPH & 209 & 21.4 & 0.10 \\
\hline & Kidney & Cytosol & NADPH & 94.2 & 9.01 & 0.10 \\
\hline
\end{tabular}

only at high concentrations (Supplemental Fig. S2). A comparison of the NADPH-supported reduction of $\mathbf{2}$ in cytosolic fractions across human tissues in terms of $\mathrm{Cl}_{\text {int }}$ resulted in a rank order of activity as follows: intestine $>$ liver $>$ lung $\approx$ kidney (Table 1$)$. Figure 6 shows $\mathrm{Cl}_{\text {int }}$ data determined by the disappearance of $2(1 \mu \mathrm{M})$ in NADPH-fortified cytosolic fractions from the liver, intestine, kidney, and lung from humans, cynomolgus monkeys, dogs, and rats. In general, cynomolgus monkeys and dogs exhibited similar activity to that of humans across tissues. However, rats displayed significantly less activity across all matrices.

Enzyme-Identification Studies. Currently, highly selective inhibitors for most carbonyl-reducing enzymes are not available. Therefore, identifying the enzyme(s) responsible for the conversion of $\mathbf{2}$ to $\mathbf{1}$ was undertaken using a set of inhibitors which are known to inhibit one or more carbonyl-reducing enzymes. Inhibition of the conversion of $\mathbf{2}$ to $\mathbf{1}$ was determined using HLCyt supplemented with NADPH. Inhibition as a percentage of control was determined at 10 and $100 \mu \mathrm{M}$ inhibitor concentrations for the inhibitors listed in Table 2. At the $10 \mu \mathrm{M}$ inhibitor concentration, $<20 \%$ inhibition was observed for all inhibitors except for menadione and quercetin, which inhibited $32.6 \%$ and $56.4 \%$, respectively. At the $100 \mu \mathrm{M}$ inhibitor concentration, menadione, quercetin, ethacrynic acid, and disulfiram showed 95.7\%, 81.6\%, 34.4\%, and $43.5 \%$ inhibition, respectively. Based on the reported inhibition of carbonyl-reducing enzymes, these results indicate the involvement of a CBR or a member of the SDR.

\section{Discussion}

The identification of $\mathbf{1}$ as a potent and selective inhibitor of aldosterone synthase (CYP11B2) led to characterization of its absorption, distribution, metabolism, and excretion in both in vitro systems and in vivo in preclinical species. In these systems, $\mathbf{1}$ was characterized as a

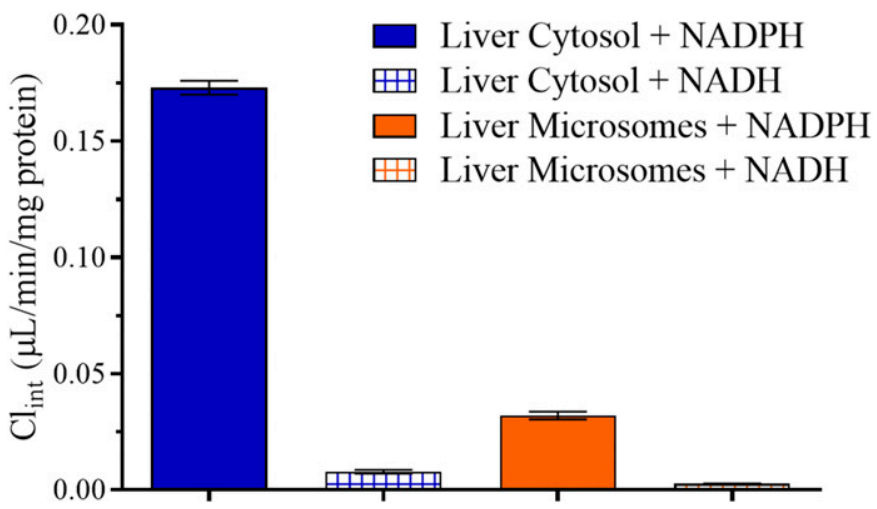

Fig. 5. Comparison of the reductive metabolism of $\mathbf{2}$ to $\mathbf{1}$ in human liver cytosol and microsomes supplemented with NADPH or NADH. low-clearance compound $(<2.5 \mathrm{ml} / \mathrm{min} / \mathrm{kg}$, data not included). Although 1 was found to be metabolically stable, sufficient turnover of 1 was observed to allow for the identification of metabolites, as shown in Fig. 2. In rP450s, human LM, and human hepatocytes, three monooxidation metabolites were observed with $\mathrm{m} / \mathrm{z}$ 333. One of these metabolites was confirmed to be the pyridine $N$-oxide metabolite (4). Another of these metabolites was determined to be hydroxylated on the methylene adjacent to the urea using the deuterated analog $\mathbf{5}$, resulting in the ring-intact hydroxyl species and/or the ring-opened aldehyde metabolite. Subsequent reduction and oxidation of the aldehyde form of this metabolite likely gives rise to both the $\mathrm{m} / \mathrm{z}, 334$ and $\mathrm{m} / \mathrm{z}$, 348 metabolites, respectively. As formation of these metabolites likely requires cytosolic alcohol and aldehyde dehydrogenases, respectively, these metabolites are only observed in incubations of $\mathbf{1}$ with hepatocytes. The specific position of the third mono-oxidation metabolite was not determined. A minor ketone metabolite, 2 ( $<1 \%$ of parent based on UV in HLM and hepatocytes), was confirmed by synthesis of an authentic standard. Subsequently, 2 was shown to possess inhibitory activity against CYP11B2 and a high degree of selectivity relative to CYP11B1. Due to its activity against the pharmacological target as well as potential for reversible metabolism contributing to the low clearance of $\mathbf{1}$, studies to determine enzymes responsible for the formation and subsequent metabolism of this ketone metabolite were undertaken.

As both NADH and NADPH are capable of supporting reductive metabolism depending on the enzyme(s) involved (Rosemond and Walsh, 2004), we initially sought to determine the optimal cofactor and matrix for reduction of $\mathbf{2}$ to $\mathbf{1}$ in HLM and HLCyt. In HLMs, both oxidative metabolism and reduction of $\mathbf{2}$ to $\mathbf{1}$ were shown to be minor. However, $\mathbf{2}$ was found to be metabolized rapidly via reduction in HLCyt

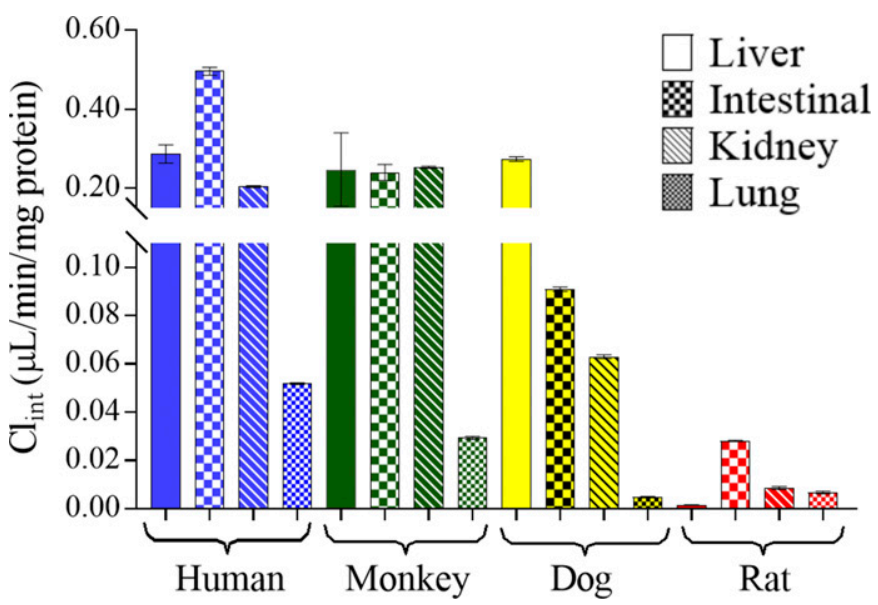

Fig. 6. Comparison of the reductive metabolism of $\mathbf{2}$ in cytosols across tissues from humans, monkeys, dogs, and rats supplemented with NADPH. 
TABLE 2

The effect of reductase inhibitors on the conversion of $\mathbf{2}$ to $\mathbf{1}$ in HLCyt supplemented with NADPH

\begin{tabular}{|c|c|c|c|c|}
\hline \multirow{2}{*}{ Inhibitor } & \multirow{2}{*}{ Enzyme Targets } & \multicolumn{2}{|c|}{$\%$ Inhibition } & \multirow{2}{*}{ Reference } \\
\hline & & $10 \mu \mathrm{M}$ & $100 \mu \mathrm{M}$ & \\
\hline Phenobarbital & $\begin{array}{l}\text { AKR1A1 } \\
\text { AKR1B1 }\end{array}$ & 2.4 & -2.7 & Gebel and Maser (1992) \\
\hline Zopolrestat & $\begin{array}{l}\text { AKR1B1 } \\
\text { AKR1C1 } \\
\text { AKR1C2 }\end{array}$ & $\begin{array}{r}10.3 \\
6.6\end{array}$ & $\begin{array}{r}15.2 \\
6.0\end{array}$ & $\begin{array}{l}\text { Mylari et al. (1991) } \\
\text { Atalla et al. (2000), Steckelbroeck et al. (2006) }\end{array}$ \\
\hline Phenolphthalein & $\begin{array}{l}\text { AKR1C3 } \\
\text { AKR1C4 } \\
\text { AKR1C1 } \\
\text { AKR1C2 }\end{array}$ & 8.0 & 18.1 & Matsuura et al. (1997), Atalla et al. (2000) \\
\hline Flufenamic acid & $\begin{array}{l}\text { AKR1C3 } \\
\text { AKR1C4 }\end{array}$ & & & \\
\hline Chenodeoxycholic acid & $\begin{array}{l}\text { AKR1C2 } \\
\text { AKR1C1 }\end{array}$ & $\begin{array}{r}-0.9 \\
5.0\end{array}$ & $\begin{array}{r}-2.9 \\
6.4\end{array}$ & $\begin{array}{l}\text { Bauman et al. (2005) } \\
\text { Hara et al. (1990) }\end{array}$ \\
\hline Medroxyprogesterone 17-acetate & $\begin{array}{l}\text { AKR1C2 } \\
\text { AKR1C4 }\end{array}$ & & & \\
\hline Dexamethasone & $\begin{array}{l}\text { AKR1C4 } \\
\text { AKR1C1 } \\
\text { AKR1C2 }\end{array}$ & $\begin{array}{r}0.7 \\
-0.2\end{array}$ & $\begin{array}{r}-7.6 \\
12.2\end{array}$ & $\begin{array}{l}\text { Deyashiki et al. (1992) } \\
\text { Gebel and Maser (1992) }\end{array}$ \\
\hline Indomethacin & $\begin{array}{c}\text { AKR1C3 } \\
\text { AKR1C4 } \\
\text { CBR }\end{array}$ & & & \\
\hline Menadione & CBR/SDR & 32.6 & 95.7 & Wermuth (1981), Porter et al. (2000) \\
\hline Quercetin & CBR/SDR & 56.4 & 81.6 & Wermuth (1981) \\
\hline Ethacrynic acid & $\mathrm{CBR} / \mathrm{SDR}$ & 11.6 & 34.4 & $\begin{array}{l}\text { Wermuth (1981), Atalla et al. (2000), Atalla } \\
\text { and Maser (2001) }\end{array}$ \\
\hline Dicumarol & $\begin{array}{c}\text { Quinone } \\
\text { oxidoreductase }\end{array}$ & 19.7 & 11.2 & Edwards et al. (1980) \\
\hline 4-Methyl pyrazole & $\begin{array}{c}\text { Alcohol } \\
\text { dehydrogenase }\end{array}$ & 1.8 & 2.2 & Atalla and Maser (2001) \\
\hline Disulfiram & $\begin{array}{c}\text { Aldehyde } \\
\text { dehydrogenase }\end{array}$ & 5.9 & 43.5 & Kraemer and Deitrich (1968) \\
\hline
\end{tabular}

Incubations were performed at a $15 \mu \mathrm{M}$ concentration of 2 and a $1 \mathrm{mg} / \mathrm{ml}$ protein concentration of HLCyt for 5 minutes at $37^{\circ} \mathrm{C}$.

supplemented with NADPH. Analysis of samples of the cytosolic incubation indicated that the reduction of $\mathbf{2}$ occurred stereoselectively back to 1 and not to its enantiomer, 3 (Fig. 4). Such stereoselectivity for carbonyl reduction is not without precedent and has been reported for ketotifen (Breyer-Pfaff and Nill, 2000), nortriptyline (Breyer-Pfaff and Nill, 2000), and dolasetron (Breyer-Pfaff and Nill, 2004). Furthermore, the reduction of $\mathbf{2}$ to $\mathbf{1}$ in human cytosol fractions across a number of tissues (Fig. 5; Supplemental Table S4) was supported predominantly by NADPH with considerably less reductase activity observed in the presence of NADH.

The NADPH-catalyzed reduction of $\mathbf{2}$ to $\mathbf{1}$ exhibited comparable $\mathrm{K}_{\mathrm{m}}$ values across tissues and in recombinant CBR1 (Table 1), although $\mathrm{V}_{\max }$ values varied considerably across tissues. In terms of $\mathrm{Cl}_{\text {int }}$, the cytosolic reduction activity was found to increase in rate across human tissues (Table 1 ) in the following order: intestine $>$ liver $>$ kidney $\approx$ lung. However, some caution must be taken in the determined kinetic parameters (i.e., $\mathrm{K}_{\mathrm{m}}, \mathrm{V}_{\mathrm{max}}$, and $\mathrm{Cl}_{\mathrm{int}}$ ), as the reported $\mathrm{K}_{\mathrm{m}}$ values in most matrices approach the solubility limit of $\mathbf{1}$.

Akin to human, reduction of $\mathbf{2}$ was also observed in cytosolic fractions from other preclinical species. Similar reductive activities were observed across tissues in humans, monkeys, and dogs. However, reductive metabolism across tissues from rats was considerably slower than in human tissues. These observations are consistent with known differences in the function and expression of CBR1 across preclinical species and humans. These differences have been described in a recent publication which evaluated the tissue distribution of CBR1 across preclinical species and reported that: 1) cbr1 is not expressed in rat liver, 2) cbr1 demonstrates greater specificity in steroid reduction, and 3) cbr1 is highly expressed in reproductive tissues (Shi and Di, 2017).

To provide insights into the enzyme(s) responsible for the reduction of 2, a set of inhibition studies were undertaken. As selective inhibitors for many of the carbonyl reductase enzymes have not been identified, a set of inhibitors targeting one or more reductase enzymes were used at 10 and $100 \mu \mathrm{M}$ concentrations. The set of inhibitors are presented in Table 2 and include phenobarbital (AKR1A1, AKR1B1), zopolrestat (AKR1B1), phenolphthalein (AKR1C1, AKR1C2, AKR1C3, AKR1C4), flufenamic acid (AKR1C1, AKR1C2, AKR1C3, AKR1C4), chenodeoxycholic acid (AKR1C2), medroxyprogesterone 17-acetate (AKR1C1, AKR1C2, AKR1C4), dexamethasone (AKR1C4), indomethacin (AKR1C1, AKR1C2, AKR1C3, AKR1C4, carbonyl reductase), menadione (CR/SDR), quercetin (CR/SDR), ethacrynic acid (CR/SDR), dicumarol (quinone oxidoreductase), 4-methyl pyrazole (alcohol dehydrogenase), and disulfiram (aldehyde dehydrogenase). As shown in Table 2, significant inhibition was observed for menadione (96\% inhibition), quercetin ( $82 \%$ inhibition), 
disulfiram (44\% inhibition), and ethacrynic acid (34\% inhibition) at a $100 \mu \mathrm{M}$ inhibitor concentration. Likewise, both quercetin and menadione also showed significant inhibition at a $10 \mu \mathrm{M}$ inhibitor concentration. Inhibition by menadione, quercetin, and ethacrynic acid indicates that a CR/SDR enzyme such as CBR1 is responsible for the reduction of 2. This is further supported by the location of the reductase in the cytosolic fraction and the greater rate of metabolism with incubations supplemented with NADPH rather than NADH. Inhibition of reduction of $\mathbf{2}$ by the aldehyde dehydrogenase inhibitor disulfiram can be explained by a lack of selectivity for this inhibitor at higher concentrations. Additionally, disulfiram has been previously shown to inhibit two carbonyl reductases isolated from rat ovaries (Iwata et al., 1992).

Data obtained from inhibition studies, the greater activity observed in the presence of NADPH versus NADH, studies with recombinant CBR1, and the majority of reductase activity observed in the cytosolic fraction together indicate that CBR1 is the enzyme responsible for this transformation in humans, and that the reduction reaction is selective for CBR1. The reductive activity toward $\mathbf{2}$ observed across human tissues also appears to be in agreement with the protein-expression profile of CBR1 reported by Hua et al. (2017).

Although many compounds have been identified as substrates for CBR1, the selectivity of most toward other carbonyl-reducing enzymes and cytochrome $\mathrm{P} 450 \mathrm{~s}$ is generally poor. Additionally, many of the probe substrates are small, low-molecular-weight compounds with limited utility for MS detection. The selective reduction of $\mathbf{2}$ by CBR1, the relatively slow oxidation of $\mathbf{1}$ to $\mathbf{2}$ by P450 enzymes, the lack of inhibitory potency of 2 against P450 enzymes (other than CYP11B2), and the favorable MS characteristics for this compound make $\mathbf{2}$ a useful probe substrate to assess CBR1 activity in vitro in both subcellular factions and in cell-based systems.

In addition to the identification of a potentially useful probe of CBR1, the in vitro characterization of the metabolic interconversion of $\mathbf{1}$ and $\mathbf{2}$ alleviated concerns regarding inhibitory potency of $\mathbf{2}$ toward CYP11B2, the stereoselectivity of the reductive metabolism of $\mathbf{2}$, and the contribution of the reversible metabolism of $\mathbf{2}$ contributing to the low clearance of 1. In vitro studies indicated that serendipitous conversion of $\mathbf{2}$ back to $\mathbf{1}$ occurred. The rate of back-reduction of $\mathbf{2}$ to $\mathbf{1}$ is anticipated to result in insignificant circulating levels of $\mathbf{2}$ contributing to the pharmacology. Additionally, the minimal formation of $\mathbf{2}$ in systems lacking CBR1 suggests that oxidative metabolism of $\mathbf{1}$ is slow and indicates that reversible metabolism involving $\mathbf{2}$ is likely minimal.

\section{Acknowledgments}

We thank Xin Guo, Kenneth Meyers, Bryan McKibben, and John Lord for their synthesis of compounds $\mathbf{1}-\mathbf{5}$.

\section{Authorship Contributions}

Participated in research design: Arenas, Frederick, Cerny.

Conducted experiments: Arenas, Frederick.

Performed data analysis: Arenas, Frederick, Cerny.

Wrote or contributed to the writing of the manuscript: Ramsden, Smith, Frederick, Cerny.

\section{References}

Atalla A, Breyer-Pfaff U, and Maser E (2000) Purification and characterization of oxidoreductasescatalyzing carbonyl reduction of the tobacco-specific nitrosamine 4-methylnitrosamino-1-(3pyridyl)-1-butanone (NNK) in human liver cytosol. Xenobiotica 30:755-769.

Atalla A and Maser E (2001) Characterization of enzymes participating in carbonyl reduction of 4-methylnitrosamino-1-(3-pyridyl)-1-butanone (NNK) in human placenta. Chem Biol Interact 130-132:737-748.

Balestra M, Burke J, Chen Z, Cogan D, Fader L, Guo X, McKibben B, Marshall DR, Nemoto PA, and $\mathrm{Yu} H$ (2014) inventors, Boehringer Ingelheim International GmbH, assignee. Naphthyridines, azaindoles and related compounds as aldosterone synthase inhibitors and their preparation Patent Number US20140323468A1. 2014-10-30, Boehringer Ingelheim International GmbH, Germany.

Bauman DR, Rudnick SI, Szewczuk LM, Jin Y, Gopishetty S, and Penning TM (2005) Development of nonsteroidal anti-inflammatory drug analogs and steroid carboxylates selective for human aldo-keto reductase isoforms: potential antineoplastic agents that work independently of cyclooxygenase isozymes. Mol Pharmacol 67:60-68.

Boldyreff B and Wehling M (2003) Non-genomic actions of aldosterone: mechanisms and consequences in kidney cells. Nephrol Dial Transplant 18:1693-1695.

Brem AS and Gong R (2015) Therapeutic targeting of aldosterone: a novel approach to the treatment of glomerular disease. Clin Sci (Lond) 128:527-535.

Breyer-Pfaff U and Nill K (2000) High-affinity stereoselective reduction of the enantiomers of ketotifen and of ketonic nortriptyline metabolites by aldo-keto reductases from human liver. Biochem Pharmacol 59:249-260.

Breyer-Pfaff U and Nill K (2004) Carbonyl reduction of naltrexone and dolasetron by oxidoreductases isolated from human liver cytosol. J Pharm Pharmacol 56:1601-1606.

Cerny MA (2013) Progress towards clinically useful aldosterone synthase inhibitors. Curr Top Med Chem 13:1385-1401.

Cerny MA (2016) Prevalence of non-cytochrome P450-mediated metabolism in Food and Drug Administration-Approved Oral and Intravenous Drugs: 2006-2015. Drug Metab Dispos 44: $1246-1252$.

Cerny MA, Csengery A, Schmenk J, and Frederick K (2015) Development of CYP11B1 and CYP11B2 assays utilizing homogenates of adrenal glands: utility of monkey as a surrogate for human. J Steroid Biochem Mol Biol 154:197-205.

Deyashiki Y, Taniguchi H, Amano T, Nakayama T, Hara A, and Sawada H (1992) Structural and functional comparison of two human liver dihydrodiol dehydrogenases associated with 3 alphahydroxysteroid dehydrogenase activity. Biochem J 282:741-746.

Dooley R, Harvey BJ, and Thomas W (2012) Non-genomic actions of aldosterone: from receptors and signals to membrane targets. Mol Cell Endocrinol 350:223-234.

Edwards YH, Potter J, and Hopkinson DA (1980) Human FAD-dependent NAD(P)H diaphorase. Biochem J 187:429-436.

Funder JW (2001) Non-genomic actions of aldosterone: role in hypertension. Curr Opin Nephrol Hypertens 10:227-230.

Gebel T and Maser E (1992) Characterization of carbonyl reducing activity in continuous cell lines of human and rodent origin. Biochem Pharmacol 44:2005-2012.

Hara A, Taniguchi H, Nakayama T, and Sawada H (1990) Purification and properties of multiple forms of dihydrodiol dehydrogenase from human liver. J Biochem 108:250-254.

Hu Q, Yin L, and Hartmann RW (2014) Aldosterone synthase inhibitors as promising treatments for mineralocorticoid dependent cardiovascular and renal diseases. J Med Chem 57:5011-5022.

Hua W, Zhang H, Ryu S, Yang X, and Di L (2017) Human tissue distribution of carbonyl reductase 1 using proteomic approach with liquid chromatography-tandem mass spectrometry. J Pharm Sci 106: $1405-1411$.

Hwang MH, Yoo JK, Luttrell M, Kim HK, Meade TH, English M, Segal MS, and Christou DD (2013) Mineralocorticoid receptors modulate vascular endothelial function in human obesity. Clin Sci (Lond) 125:513-520.

Iwata N, Inazu N, and Satoh T (1992) Consecutive treatment of disulfiram inhibits ovarian carbonyl reductase activity in rats. Jpn J Pharmacol 58:167-173.

Kraemer RJ and Deitrich RA (1968) Isolation and characterization of human liver aldehyde dehydrogenase. J Biol Chem 243:6402-6408.

Malátková P and Wsól V (2014) Carbonyl reduction pathways in drug metabolism. Drug Metab Rev 46:96-123.

Matsuura K, Deyashiki Y, Sato K, Ishida N, Miwa G, and Hara A (1997) Identification of amino acid residues responsible for differences in substrate specificity and inhibitor sensitivity between two human liver dihydrodiol dehydrogenase isoenzymes by site-directed mutagenesis. Biochem J 323:61-64.

Mylari BL, Larson ER, Beyer TA, Zembrowski WJ, Aldinger CE, Dee MF, Siegel TW, and Singleton DH (1991) Novel, potent aldose reductase inhibitors: 3,4-dihydro-4-oxo-3-[[5(trifluoromethyl)-2-benzothiazolyl] methyl]-1-phthalazineacetic acid (zopolrestat) and congeners. J Med Chem 34:108-122.

Oparil S and Schmieder RE (2015) New approaches in the treatment of hypertension. Circ Res 116: 1074-1095.

Porter SJ, Somogyi AA, and White JM (2000) Kinetics and inhibition of the formation of 6betanaltrexol from naltrexone in human liver cytosol. Br J Clin Pharmacol 50:465-471.

Rosemond MJ and Walsh JS (2004) Human carbonyl reduction pathways and a strategy for their study in vitro. Drug Metab Rev 36:335-361.

Shi SM and Di L (2017) The role of carbonyl reductase 1 in drug discovery and development. Expert Opin Drug Metab Toxicol 13:859-870.

Steckelbroeck S, Oyesanmi B, Jin Y, Lee SH, Kloosterboer HJ, and Penning TM (2006) Tibolone metabolism in human liver is catalyzed by 3 alpha/3beta-hydroxysteroid dehydrogenase activities of the four isoforms of the aldo-keto reductase (AKR)1C subfamily. J Pharmacol Exp Ther 316: $1300-1309$.

Vinson GP and Coghlan JP (2010) Expanding view of aldosterone action, with an emphasis on rapid action. Clin Exp Pharmacol Physiol 37:410-416.

Weldon SM, Cerny MA, Gueneva-Boucheva K, Cogan D, Guo X, Moss N, Parmentier JH, Richman JR, Reinhart GA, and Brown NF (2016) Selectivity of BI 689648, a novel, highly selective aldosterone synthase inhibitor: comparison with FAD286 and LCI699 in nonhuman primates. J Pharmacol Exp Ther 359:142-150.

Wermuth B (1981) Purification and properties of an NADPH-dependent carbonyl reductase from human brain. Relationship to prostaglandin 9-ketoreductase and xenobiotic ketone reductase. $J$ Biol Chem 256:1206-1213.

Address correspondence to: Matthew A. Cerny, Pharmacokinetics, Dynamics and Metabolism, Pfizer, Inc., Eastern Point Rd., Groton, CT 06340. E-mail: matthew.cerny@pfizer.com 